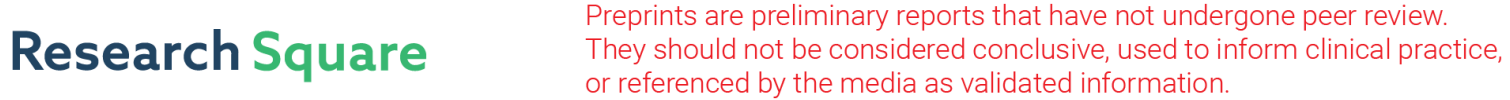 \\ The Cross-Industry Universal Laws of Exponential Booking Curves
}

Masaru Shintani ( $\square$ masaru_shintani@forcia.com )

Kyoto University

Ken Umeno

Kyoto University https://orcid.org/0000-0002-9162-1261

\section{Physical Sciences - Article}

Keywords: universal laws, booking curve, exponential decay, economic indicators

Posted Date: September 28th, 2021

DOI: https://doi.org/10.21203/rs.3.rs-941798/v1

License: (9) This work is licensed under a Creative Commons Attribution 4.0 International License. Read Full License 


\title{
The Cross-Industry Universal Laws of Exponential Booking Curves
}

\author{
Masaru Shintani ${ }^{1,2^{*}}$ and Ken Umeno ${ }^{1 \dagger}$ \\ $1^{*}$ Department of Applied Mathematics and Physics, Graduate \\ School of Informatics, Kyoto University, Yoshida Honmachi, \\ Sakyo-ku, 606-8501, Kyoto, Japan. \\ ${ }^{2}$ Corporate Development Department, FORCIA, Inc., JR \\ SHINJUKU MIRAINA TOWER 13F, 1-6, Shinjuku 4 chome, \\ Shinjuku-ku, 160-0022, Tokyo, Japan.
}

*Corresponding author(s). E-mail(s): shintani.masaru.28a@st.kyoto-u.ac.jp; Contributing authors: umeno.ken.8z@kyoto-u.ac.jp; ${ }^{\dagger}$ These authors contributed equally to this work.

\begin{abstract}
In recent years, the e-commerce market has grown with the spread of the internet worldwide every year [1]. Accordingly, in service industries, purchasing products with reservations has become common. With the spread of online reservations, the booking curve, which is the concept of the time series in the cumulative number of reservations and has been used for sales optimization in the airline ticket and hotel industries, has been used in various industries [2-4]. Booking curves in specific industries have been studied, but a universally applicable model across various industries has not been developed [5-8]. Here we show that booking curves can be modeled universally by the exponential decay function, and we also show that the model is valid by using real data from some industries before and after the COVID-19 pandemic, that is, under completely different market conditions. The cross-industry exponential laws of booking curves constitute an important discovery in regard to mathematical laws in the social sciences and can be applied to give leading microeconomic indicators.
\end{abstract}

Keywords: universal laws, booking curve, exponential decay, economic indicators 


\section{Methods}

We define a booking curve with a sales deadline that represents the cumulative number of reservations as follows:

$$
X(t) \stackrel{\text { def }}{=} \int_{t}^{\infty} q(s) d s,
$$

where $q(s)$ is the number of reservations $s$ days before deadlines. Then, we obtain averaged booking curves (ABCs) for time series $\{X(t ; n) \mid n=$ $1,2, \ldots, N\}$ for $N$ days as follows: $\mathbb{E}[X(t ; n)]=\frac{1}{N} \sum_{n=1}^{N} X(t ; n)$. On the other hand, we model the $\mathrm{ABC} \mathbb{E}[X(t)]$ with the following differentiable and monotonous non-increasing function $\phi$ :

$$
\mathbb{E}[X(t)]=A \phi\left(t ; \beta_{t}\right),
$$

where $A(>0)$ is a capacity constant calculated by the scale of the facility or the magnitude of demand and $\beta_{t}>0$ is an environmental variable given by the timing of when users make reservations and represents the extent of the increase (decrease) in the number of reservations over time ( $t$ increases). Then, the function $\phi\left(t, \beta_{t}\right)$ satisfies the following properties:

$$
\begin{aligned}
& \phi\left(0 ; \beta_{0}\right)=1, \lim _{t \rightarrow \infty} \phi\left(t ; \beta_{t}\right)=0, \\
& \phi\left(t ; \beta_{t}\right) \geq \phi\left(t+1 ; \beta_{t+1}\right) \text { for } \forall t \geq 0 .
\end{aligned}
$$

Here, we set the following assumptions. It is assumed that the environmental variable in the facility is constant; that is, the parameter satisfies $\beta_{t}=\beta$ (= constant $)$. This means that the environment surrounding the facility (for example, the market environment) is constant during the reservation period, and we call this assumption the uniform demand bath assumption (UDBA), which is associated with the heat bath in thermodynamics [9].

Under this assumption, the ABC of facility $F_{1}$ is expressed by Eq. (2). Considering the state of facility $\mathrm{F}_{1}$, going back by $\delta t$ infinitesimal times, the number of reservations at time $\delta t$ is $A \phi(\delta t ; \beta)=A^{\prime}$. Then, we consider a virtual facility, $\mathrm{F}_{2}$, with a capacity constant, $A^{\prime}$. Since the time $\delta t$ is infinitesimal, it is reasonable that the environmental variable in $\mathrm{F}_{2}$ is equal to $\beta$, which is the same as in $\mathrm{F}_{1}$. Here, the number of reservations in the state of going back by time $t_{1}$ for the $\mathrm{ABC}$ of $\mathrm{F}_{2}$ is obtained as $A^{\prime} \phi\left(t_{1} ; \beta\right)=A \phi(\delta t ; \beta) \phi\left(t_{1} ; \beta\right)$. On the other hand, this value is equivalent to the number of reservations $A \phi\left(t_{2} ; \beta\right)$ when the time is traced back forward to $\delta t+t_{1}\left(\equiv t_{2}\right)$ in $\mathrm{F}_{1}$. Therefore, we obtain the following equation:

$$
\begin{aligned}
A \phi\left(t_{2} ; \beta\right) & =A \phi(\delta t ; \beta) \phi\left(t_{1} ; \beta\right) \\
t_{2} & =\delta t+t_{1} .
\end{aligned}
$$


From Eqs. (3) and (4), function $\phi$ is uniquely determined as $\phi(t ; \beta)=$ $\exp (-\beta t)$ (see supplemental information 4 for an explanation), and we obtain the exponential laws for ABCs as $\mathbb{E}[X(t)]=A \exp (-\beta t)$.

Here, we investigate two issues to verify the universality of our model. First, we study whether some actual data fit into the following approximate expression:

$$
\mathbb{E}[X(t ; n)] \simeq A \exp (-\beta t) .
$$

Second, we focus on the reasonability of the UDBA by using data from before and after the COVID-19 pandemic period that have a large fluctuation in the magnitude of demand. For each analysis, we use two years of data from 2019 to 2020 for three facilities in the hotel and car rental industries. The details of the data and facilities are described in supplemental information 6 .

\section{Results}

Fig. 1 illustrates the results based on fitting real data into Eq. (5). First, we investigate the exponential laws of ABCs in terms of the cross-industry, crossfacility, and cross-condition of the market and confirm that real data are almost generally expressed by exponential functions from each perspective. Furthermore, the difference between the environmental variables $\beta$ across industries or facilities, which are the parameters given by the timing of when people make reservations, results in remarkable differences in products such as resorts and business hotels. Second, regarding the reasonability of the UDBA, Fig. 2 represents the relationship between $M S E$ and $C V_{\tau}$, each of which is defined as an error based on each fitting exponential function and an indicator of the magnitude of environmental fluctuation. It is confirmed that contents that have large $M S E$ also have large $C V_{\tau}$; therefore, this means that the market environment is not stable when ABCs cannot follow exponential laws. As the contraposition of the exponential laws of ABCs under the UDBA, this result also supports the reasonability of our model.

\section{Discussions}

In the real world, exponential laws have been universally observed in various systems (natural sciences, social sciences, statistical physics, etc.) [9, 12-15]. It is also known that as another universal law, there is a statistical law, such as the super generalized central limit theorem based on the power law $[16,17]$, which is a ubiquitous characteristic found in such systems. In the present case, considering the generality based on Eqs. (2), (3) and (4), we expect that the exponential laws of ABCs can be universally established in industries over those introduced in the Results section.

Furthermore, parameter $A$, which is derived from the magnitude of demand in the model, is expected to give a leading economic indicator. Fig. 3 illustrates the service industry purchasing managers ' index (PMI) in Japan (see 
supplemental information 7 for a description of the PMI) and indicator parameter $A$ based on the exponential model of the ABCs for each month. The PMI is a macroeconomic indicator based on many questionnaires administered to the entire domestic service industry $[18,19]$, while parameter $A$ is obtained directly from real data for each facility. Fig. 3 confirms that the value of $A$ has changed, especially prior to the emergency declaration period from April 2020 and the tourism campaign period from July of the same year. Meanwhile, indicator $A$ plays a role in demand forecasting for the facility; in addition, the model has more general availability, for example, regarding the area and industry. Hence, we expect the model to serve as an economic model that gives leading microeconomic indicators.

\section{Supplementary information.}

\section{Proof of leading $\phi(t ; \beta)$ into exponential decay functions}

We show that a differentiable function $\phi$, which satisfies the following Eqs. (6) and (7) under the UDBA in the main paper, is derived uniquely into an exponential decay function.

$$
\begin{gathered}
\phi(0 ; \beta)=1, \lim _{t \rightarrow \infty} \phi(t ; \beta)=0, \\
\phi(t ; \beta) \geq \phi(t+1 ; \beta) \text { for } \forall t \geq 0 . \\
\begin{array}{c}
A \phi\left(t_{2} ; \beta\right)=A \phi(\delta t ; \beta) \phi\left(t_{1} ; \beta\right) \\
t_{2}=\delta t+t_{1}
\end{array}
\end{gathered}
$$

We obtain the following equation for $\phi$ independent of $A$ since Eq. (2) is reasonably satisfied for any $t_{1}$ or $\delta t$ under the UDBA:

$$
\phi\left(\delta t+t_{1} ; \beta\right)=\phi(\delta t ; \beta) \phi\left(t_{1} ; \beta\right) \text { for } \forall \delta t, t_{1}>0 .
$$
$\lim _{\delta t \rightarrow 0}$.

Accordingly, we obtain the following identity equation for $\phi$ for any $t$ and $\delta t$ with the criterion $\phi(0)=1$ from Eq. (6).

$$
\phi(t+\delta t)-\phi(t)=\phi(t)(\phi(\delta t)-\phi(0)) .
$$

In addition, the following equation is given by dividing by $\delta t$ and applying

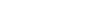

$$
\lim _{\delta t \rightarrow 0} \frac{\phi(t+\delta t)-\phi(t)}{\delta t}=\phi(t) \lim _{\delta t \rightarrow 0} \frac{\phi(\delta t)-\phi(0)}{\delta t} .
$$


Springer Nature $2021 \mathrm{~L}_{\mathrm{E}} \mathrm{T} \mathrm{X}$ template

The Cross-Industry Universal Laws of Exponential Booking Curves

\begin{tabular}{cccc} 
Facility & Acquisition period & $\begin{array}{c}\text { The number of } \\
\text { records }\end{array}$ & $\begin{array}{c}\text { Including } \\
\text { canceled records }\end{array}$ \\
\hline hotel 1 & & 141,828 & yes \\
hotel 2 & 59,075 & no \\
hotel 3 & from Jan. 1. 2019 & 23,787 & no \\
car rental 1 & to Dec. 31. 2020 & 62,401 & yes \\
car rental 2 & & 334,378 & yes \\
car rental 3 & 48,472 & yes
\end{tabular}

Table 1 Dataset information used for our verification. We acquired the reservation data of six facilities for two years. One record data represents one piece of reservation information.

Then, Eq. (11) results from regarding constant $\mathrm{C}$ as $C=\left.\frac{d \phi}{d t}\right|_{t=0}$ :

$$
\frac{d \phi}{d t}=C \phi(t)
$$

Solving this equation, we obtain function $\phi$ as follows with a certain constant $C_{0}$ :

$$
\phi(t)=\exp \left(C t+C_{0}\right) .
$$

Finally, constants $C_{0}$ and $C$, respectively, satisfy $C_{0}=0$ from $\phi(0)=1$ and $C<0$ from $\lim _{t \rightarrow \infty} \phi(t ; \beta)=0$ in Eq. (6). Thus, function $\phi$ is derived uniquely following exponential decay with a certain constant $\beta(=-C)>0$ :

$$
\phi(t)=\exp (-\beta t) .
$$

Then, the additional law that is given as Eq. (8) under a certain static environment, such as the UDBA, is also seen in the natural sciences. For example, in thermodynamics, a distribution of energy in a system with constant temperature called a heat bath satisfies a similar addition law. In our case, based on the heat bath concept, we call the criterion a demand bath with static demand environments.

\section{Dataset for verification}

Fig. 4 gives an overview of the data used for verification. The original data are published in the repository, except for information that can identify an individual or organization. We define a chartered day in the hotel 1 as having more than $50 \%$ group travel customers, which can be identified in the record data. We investigate for periods except for chartered days. All the other facilities do not have chartered days. The number of records have all of the data including chartered days. 


\begin{tabular}{ccc}
\hline \hline No. & Period & Remark for demand \\
\hline 2019 & Jan.1 - Dec.31(2019) & normal demand \\
2020 & Jan.1 - Dec.31(2020) & COVID-19 pandemic \\
$2020-1$ & Jan.1 - Mar.31(2020) & the first COVID-19 epidemic \\
$2020-2$ & Apr.1 - Jul.21(2020) & emergency declaration \\
$2020-3$ & Jul.22 - Dec.31(2020) & tourism campaign \\
\hline
\end{tabular}

Table $\overline{2}$ The information of periods divided in terms of the market conditions for our verification.

\section{Periods divided by market conditions}

Tab. 1 (This is the same as the table in Fig. 1. b in the main paper.) shows how we divide the periods to validate the exponential laws of averaged booking curves (ABCs). In 2019 and 2020, the market condition changed significantly due to the influence of the COVID-19 pandemic. Thus, we compare each of them based on annual data. In addition, in 2020, the Japanese government implemented some strategies related to the movement of people, that is, directly related to the demand environment for services such as hotels and car rentals. Therefore, we divide the periods based on these strategies. In the period from Jan. 1 to Mar. 31, COVID-19 spread in Japan for the first time; accordingly, the number of people from overseas gradually decreased. In the period from Apr. 1 to Jul. 21, the Japanese government declared a state of emergency due to COVID-19 and strongly requested citizens to self-restrict going out. In the period from Jul. 22 to Dec. 31, the government implemented the tourism campaign called "Go to Travel", which was a deep discount strategy for domestic travel products; therefore, people had more opportunities to go out.

\section{PMI: Purchasing managers' index}

The purchasing managers' index (PMI) is an economic indicator derived from a monthly survey of private companies. It is calculated based on the answers provided by companies' purchasing managers or executives regarding, for example, their firms' production, employment, inventories, order backlogs, new export orders and imports of materials and supplies. In addition, a PMI value exceeding 50 indicates better business conditions than in the previous month. Therefore, the PMI is often used as a leading economic indicator, and some economic models have been studied by using the PMI. In this study, we propose that an exponential function model of booking curves using reservation data in service industries can be applied as an economic model that gives a more microscopic and real data-driven economic leading indicator along with the PMI. The main paper introduces that the value of $A$ estimated using the exponential function model can be used as a leading economic indicator (or as one of the samples that composes it). Note that our model has three features. First, $A$ can be calculated directly from the data; second, $A$ can be calculated 
at the beginning of the month; and third, $A$ can be calculated in units smaller than the PMI, such as facilities and areas.

Acknowledgments. We thank T. Ikezawa, Y. Wakai and T. Suzuki for providing a lot of real data. This work was supported by DAIWA RESORT Co., Ltd. and some gratitude companies. We are also grateful to M. Kato, K. Sumaki, T. Yashiro and N. Natsume in FORCIA, Inc. for the execution of this project.

\section{References}

[1] Laudon, K.C., Traver, C.G.: E-commerce. Pearson, Boston, MA (2013)

[2] Lee, A.O.: Airline reservations forecasting: Probabilistic and statistical models of the booking process. Technical report, Cambridge, Mass.: Flight Transportation Laboratory, Dept. of Aeronautics and ‥(1990)

[3] Schwartz, Z., Hiemstra, S.: Improving the accuracy of hotel reservations forecasting: curves similarity approach. Journal of Travel Research 36(1), 3-14 (1997)

[4] Ma, M., Liu, J., Cao, J.: Short-term forecasting of railway passenger flow based on clustering of booking curves. Mathematical Problems in Engineering 2014 (2014)

[5] Weatherford, L.R., Kimes, S.E.: A comparison of forecasting methods for hotel revenue management. International journal of forecasting 19(3), 401-415 (2003)

[6] Popov, L.A., Romanyuk, A.V., Blinova, E.A., Gareev, R.R.: Booking curves as an instrument of increasing of independent hotel enterprise efficiency. case of russia. Journal of Environmental Management \& Tourism 8(6 (22)), 1268-1278 (2017)

[7] Tse, T.S.M., Poon, Y.T.: Analyzing the use of an advance booking curve in forecasting hotel reservations. Journal of Travel \& Tourism Marketing 32(7), 852-869 (2015)

[8] Garrow, L., Lurkin, V.: How covid-19 is impacting and reshaping the airline industry. Journal of Revenue and Pricing Management 20(1), 3-9 (2021)

[9] Lifshitz, E.M., Pitaevskii, L.P.: Statistical Physics: Theory of the Condensed State vol. 9. Elsevier, Oxford (2013)

[10] Arimura, M., Ha, T.V., Okumura, K., Asada, T.: Changes in urban mobility in sapporo city, japan due to the covid-19 emergency declarations. Transportation Research Interdisciplinary Perspectives 7, 100212 (2020) 
[11] Anzai, A., Nishiura, H.: " go to travel” campaign and travel-associated coronavirus disease 2019 cases: A descriptive analysis, july-august 2020. Journal of clinical medicine 10(3), 398 (2021)

[12] Evans, R.D., Evans, R.: The Atomic Nucleus vol. 582. McGraw-Hill, New York (1955)

[13] Vollmer, M.: Newton's law of cooling revisited. European Journal of Physics 30(5), 1063 (2009)

[14] Karagiannis, T., Le Boudec, J.-Y., Vojnović, M.: Power law and exponential decay of intercontact times between mobile devices. IEEE Transactions on Mobile Computing 9(10), 1377-1390 (2010)

[15] Shepard, R.N.: Toward a universal law of generalization for psychological science. Science 237(4820), 1317-1323 (1987)

[16] Gnedenko, B.V., Kolmogorov, A.N.: Limit Distributions for Sums of Independent Random Variables. Addison-Wesley Mathematics Series, p. 264. Addison-Wesley, Cambridge, MA (1954)

[17] Shintani, M., Umeno, K.: Super generalized central limit theorem-limit distributions for sums of non-identical random variables with power laws -. Journal of the Physical Society of Japan 87(4), 043003 (2018)

[18] Harris, E.S., et al.: Tracking the Economy with the Purchasing Managers Index. Federal Reserve Bank, New York (1991)

[19] Tsuchiya, Y.: Is the purchasing managers' index useful for assessing the economy' s strength? a directional analysis. Economics Bulletin 32(2) (2012) 

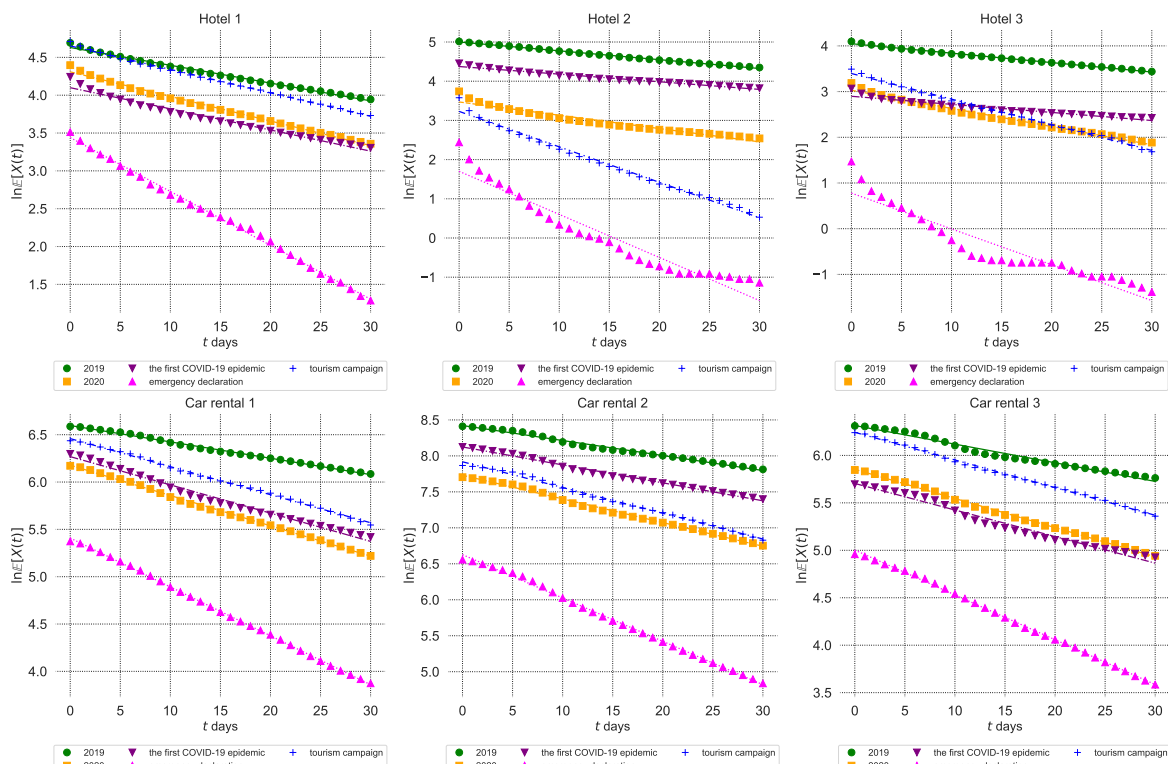

\begin{tabular}{cccc}
\hline \hline Industry & Facility region & Area type & Characteristics \\
\hline hotel 1 & Tochigi & regional city & resort hotel \\
hotel 2 & anonymous & city & business hotel
\end{tabular}

a

hotel 3

anonymous

city

car rental 1 island Ishigaki

resort

car rental 2 island Naha

resort

business hotel

car rental 3 island Miyako

resort

passenger car

passenger car

Period

Remark for demand

\begin{tabular}{cc}
\hline 2019 & Jan.1 - Dec.31(2019) \\
2020 & Jan.1 - Dec.31(2020) \\
b $2020-1$ & Jan.1 - Mar.31(2020) \\
$2020-2$ & Apr.1 - Jul.21(2020) \\
$2020-3$ & Jul.22 - Dec.31(2020)
\end{tabular}

normal demand

COVID-19 pandemic

the first COVID-19 epidemic

emergency declaration

tourism campaign

Fig. 1 The real logarithmic ABCs data with for two years, two industries and six

facilities. This figure illustrates how real data universally fit into the exponential laws with Eq. (5) in terms of three perspectives: the cross-industry, cross-facility and cross-condition of the market. We use reservation record data on six facilities in the hotel and rental car industries for validation with Eq. (5), with a fitting range of $0 \leq t \leq 30$. First, we investigate each facility in 2019 (green lines), where the market environment was relatively stable; that is, the UDBA would be satisfied. It is almost confirmed that the data follow exponentially in five facilities except car rental 3, which means that the laws are common across industries. Second, we focus on the differences in characteristics such as business and resort hotels in 2019. Although hotels 1, 2 and 3 are within the same industry, the attenuation coefficients of reservations (slopes; derived from $\beta$ ) are different; therefore, parameter $\beta$ is significantly consistent with the nature of the facility and product since it changes due to the difference in when users make reservations. Third, we compare three periods of different demand in 2020 (details in supplemental information $6[10,11]$ ), which do not necessarily satisfy the UDBA (purple, blue and pink lines). Nevertheless, in each facility, the intercept (derived from capacity constants) based on the magnitude of demand and the slopes (environment variables) differ under each period, and we find the exponential laws in some facilities and periods. 


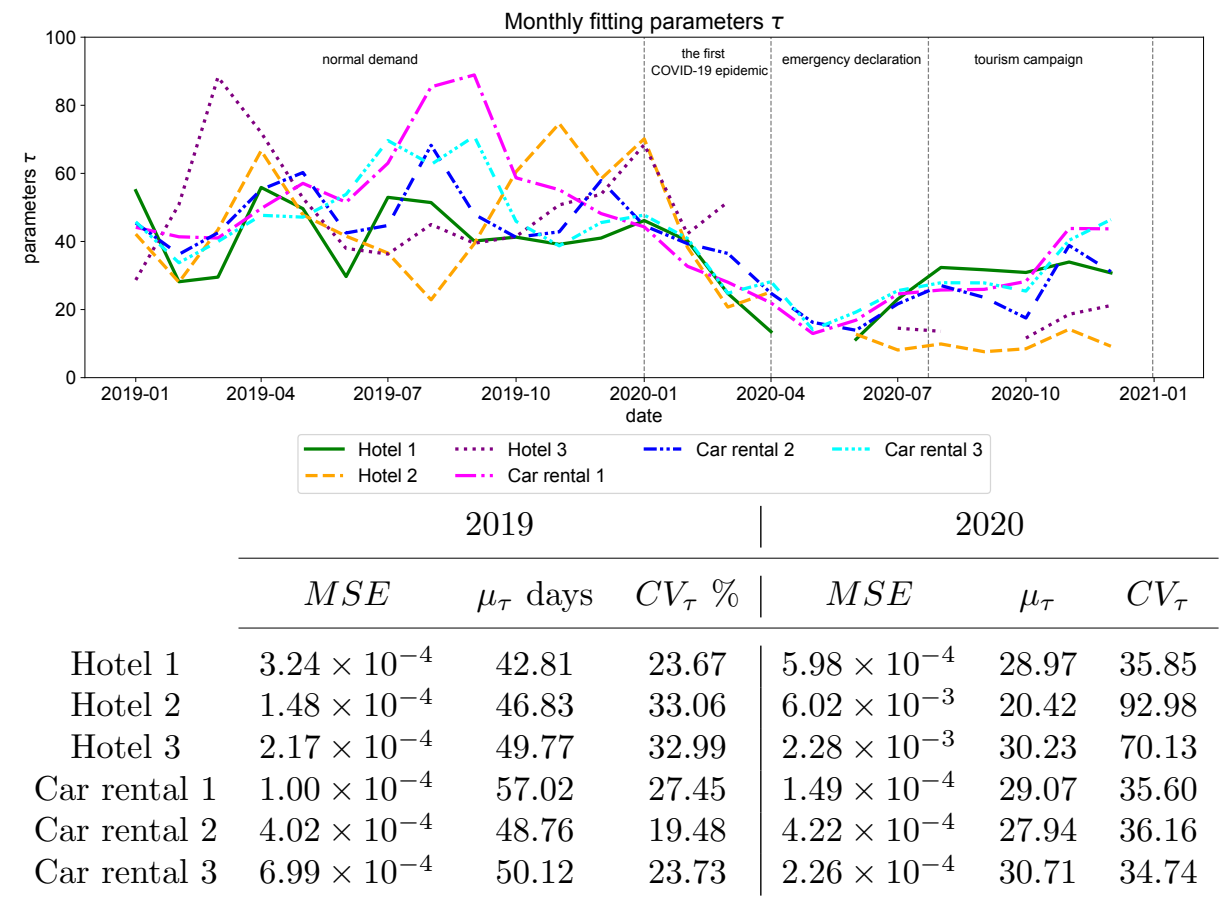

Fig. 2 The relation between the fluctuation of the environment parameter $\tau$ derived from monthly data and the error between logarithmic ABCs obtained from annual year and their regression lines. This figure illustrates how the exponential laws of ABCs relate to the environment around facilities quantitatively by using data from 2019 to 2020 . The line graph shows the transition of the time constants of exponential function $\tau_{i j}$, which is given as $\tau=1 / \beta[12]$ in Eq. (5) in year and month $i j$. The table shows the three indicators given below for 2019 and 2020. First, $M S E$ is the mean square error that represents how ABCs deviate from exponential functions as seen in green and yellow lines in Fig. 1. It is defined as follows: $\operatorname{MSE}\left(N ; t^{*}\right)=\frac{1}{t^{*}} \int_{0}^{t^{*}}|\ln (\mathbb{E}[X(s ; n)])-(\ln A-s / \tau)|^{2} d s$, where $N$ is the number of days in each period, that is, the number of booking curves $X(t)$ to be averaged; additionally, $A$ and $\tau$ are the fitting parameters obtained by Eq. (5) with $\tau=1 / \beta$. The constant $t^{*}$, which is given as $t^{*}=30$ in our observation, gives integration intervals. Second, $\mu_{\tau}$ is the annual average of $\tau_{i j}$ obtained from the ABCs in year and month $i j$; thus, it is given by $\mu_{\tau_{i}}=\underset{j}{\mathbb{E}}\left[\tau_{i j}\right]$. Third, $C V_{\tau}$ is the annual coefficient of variation of $\tau_{i j}$ [12]; thus, it is defined as follows: $C V_{\tau_{i}}=\frac{\sigma_{\tau_{i}}}{\mu_{\tau_{i}}} \times 100$, where $\sigma_{\tau_{i}}=\sqrt{\underset{j}{\mathbb{E}}\left[\left(\tau_{i j}-\mu_{\tau_{i}}\right)^{2}\right]}$. The table confirms that the $M S E$ errors in hotels 2 and 3 in 2020 have a larger order by $10^{-3}$ than other facilities and than each previous year. Furthermore, $C V_{\tau}$, which indicates the magnitude of environmental fluctuations, is large. Thus, the deviation from the exponential laws results from the instability of the environment, for example, the COVID-19 pandemic. 


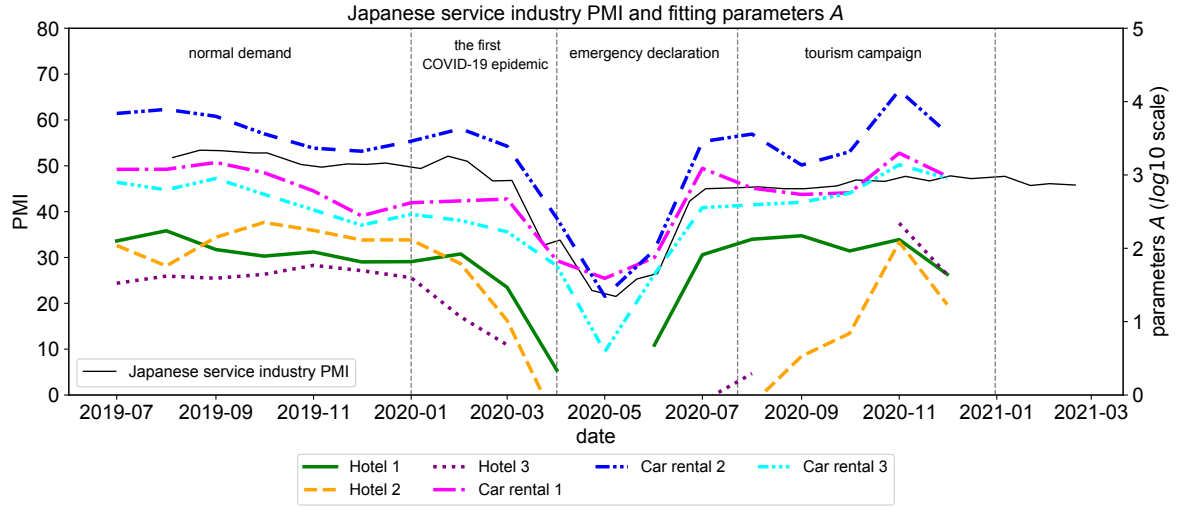

Fig. 3 The relation between Japanese service industry PMI and the estimated demand indicator $A$ in each facility at the beginning of a month. We discuss the capability of the exponential laws of ABCs as an economic model in this figure. The graph illustrates Markit's Japanese service industry PMI, which is a leading economic indicator published on markiteconomics.com, and the transition of the monthly fitting parameter $A_{i j}$ in year and month $i j$, where $30 \leq t \leq 60$ in Eq. (5). Then, since parameter $A$ is given at the beginning of the month, it can represent an indicator of the economics surrounding a facility. In fact, it is confirmed that the fitting parameters $A$ similarly decreased or increased prior to the emergency declaration period from April 2020 and the tourism campaign period from July of the same year. 